\section{Recurrent vasculitis associated with beta-haemolytic streptococcal infections}

Many agents have been incriminated in producing polyarteritis nodosa and similar systemic vasculitic disorders, including the hepatitis $B$ virus ${ }^{1}$ and various drugs. ${ }^{2} 3$ We report a case in which recurrent episodes of severe systemic vasculitis were associated with haemolytic streptococcal infections.

\section{Case report}

In 1968, when aged 17 years, the patient developed a sore throat, which worsened over four weeks despite treatment with penicillin and tetracycline. On admission to hospital she was very ill, with fever, facial oedema, swollen tongue, tender muscles, and bilateral lateral popliteal nerve palsies. A few days later she developed polyarthritis and red papules on the hands and feet. Further treatment with tetracycline, ampicillin, and erythromycin was unhelpful. Investigations showed a white cell count of $23 \times 10^{9} / 1(82 \%$ neutrophils, no eosinophils), ESR $77 \mathrm{~mm}$ in first hour, and ASO titre 230 (normal $<200$ ), and a throat swab cultured Streptococcus pyogenes. Histologically a skin biopsy specimen showed an intense arteritis. Hydrocortisone and azathioprine produced dramatic clinical improvement; after six months this was stopped and by 18 months her foot drop had resolved leaving her completely well.

She again acquired a severe sore throat in February 1977; despite treatment with erythromycin, and later tetracycline, she deteriorated and was readmitted with fever; facial oedema; gross swelling and ulceration of the tongue (see figure); painful muscles; a maculopapular rash over the buttocks;

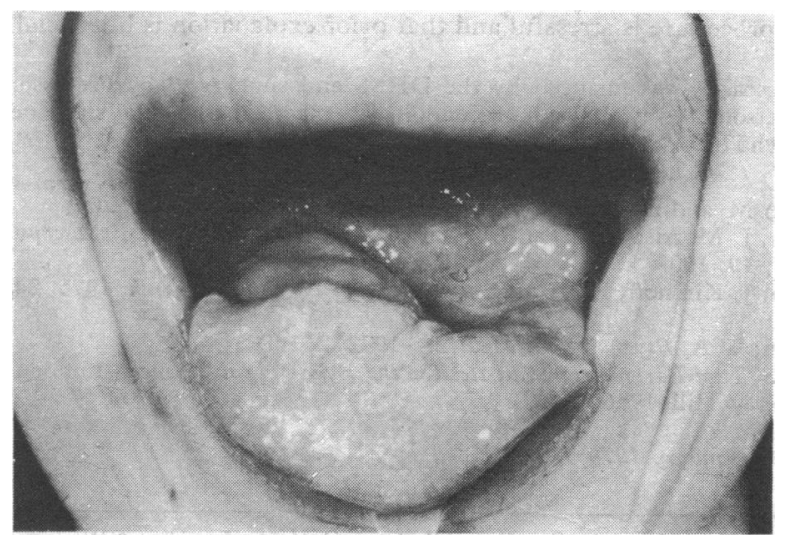

Swelling and ulceration of tongue.

radial, median, and lateral popliteal nerve palsies; and necrosis of the soft palate. The white cell count was $33.6 \times 10^{9} / 1(90 \%$ neutrophils, $1 \%$ eosinophils), ESR $103 \mathrm{~mm}$ in first hour, serum total complement 8 units (normal 30-45), C3 normal, immune complexes intermittently present, and antinuclear factor test negative; no proteinuria or haematuria; throat-swab culture negative but ASO titre above 1600 ; and chest $x$-ray film normal. A quadriceps biopsy specimen showed patchy necrosis histologically, and the tongue slough showed necrosis and an acute vasculitis. Prompt clinical improvement resulted from hydrocortisone and azathioprine, and over the next nine months progress continued with substantial resolution of the nerve palsies and some regeneration of the tongue, but $70 \mathrm{mg}$ cortisone a day is still necessary to suppress the disease.

\section{Discussion}

This patient has experienced two severe episodes of vasculitis. The clinical picture is atypical for classic polyarteritis nodosa ${ }^{4}$ or Wegener's disease. We were concerned to establish a common factor that could have triggered both illnesses, and a drug-induced arteritis was a possibility, although this phenomenon is rarer than was once sup- posed. ${ }^{5}$ Erythromycin and tetracycline were the drugs given during the early part of the 1977 episode. Erythromycin was not given in 1968 until the illness was well established, and it is not reported as causing arteritis. In 1970 the patient had a course of tetracycline for infected "spots" on her face, and after three weeks' treatment developed a few skin lesions that were possibly similar to those of 1968 . Nevertheless, in 1975 a full course of tetracycline was given with no adverse effects. There are no convincing reports incriminating this drug and the results of all our tests for drug allergy (including specific IgE and lymphocyte transformation tests) were negative. Polyarteritis is now known to be associated with the hepatitis $B$ virus in many cases, but in our patient tests for $\mathrm{HBAg}$ were negative in serum and tissue.

A relation has been noted between streptococcal infection and polyarteritis. ${ }^{4}$ Our patient had a severe sore throat as her first important symptom in both 1968 and 1977. In the first illness a haemolytic streptococcus was cultured and on both occasions the ASO titre was raised. We therefore suggest that this patient has experienced two episodes of systemic vasculitis in response to throat infections with haemolytic streptococci.

1 Gocke, D J, et al, Lancet, 1970, 2, 1149.

2 Rich, A, Bulletin of the fohns Hopkins Hospital, 1942, 71, 123.

${ }^{3}$ Citron, B P, et al, New England fournal of Medicine, 1970, 283, 1003.

${ }^{4}$ Rose, G A, and Spencer, H, Quarterly fournal of Medicine, 1957, 26, 43.

${ }^{5}$ Coombs, R R A, Gell, P G H, and Lachman, P J, Clinical Aspects of Immunology, 3rd edn, p 924. Oxford, Blackwell, 1975.

(Accepted 20 fanuary 1978)

University College Hospital and University College Hospital Medical School, London WC1

$S$ B LUCAS, BM, MRCP, lecturer in morbid anatomy

$\mathrm{J}$ MOXHAM, $\mathrm{MB}, \mathrm{MRCP}$, lecturer in medicine

\section{Spontaneous platelet aggregation reversed by flurbiprofen}

Spontaneous platelet aggregation has been associated with vascular thrombosis, particularly of the fingers and toes, which has responded to aspirin. ${ }^{1-4}$ We report the occurrence of spontaneous platelet aggregation in a patient with rubra vera who suffered from recurrent ischaemic attacks. Aspirin did not improve the clinical symptoms and had no effect on platelet aggregation. The administration of $25 \mathrm{mg}$ flurbiprofen (2-(fluoro-4-biphenylyl) propionic acid) had a dramatic effect, giving relief from symptoms within three hours.

\section{Case report}

A 63-year-old woman was first diagnosed as suffering from polycythaemia rubra vera in April 1974. The initial platelet count was $592 \times 10^{9} / 1$. She was treated with busulphan and has remained in haematological remission since. During November 1974 she first complained of intermittent diarrhoea associated with pain in the right iliac fossa. Extensive investigation failed to disclose an underlying cause but her symptoms persisted.

In March 1977 the patient reported repeated episodes of numbness of the right arm together with tingling and redness of the toes of the right foot Investigation showed a platelet count of $335 \times 10^{9} / 1$. Platelet aggregation was estimated using Born's method. ${ }^{5}$ The platelet-rich plasma $\left(320 \times 10^{9}\right.$ platelets/1) showed spontaneous aggregation four minutes after incubation at $37 \mathrm{C}$ with stirring. Spontaneous aggregation occurred when the patient's platelets were suspended in normal platelet-poor plasma but not when suspended in the patient's platelet-poor plasma. This excluded the presence of an aggregating agent in the patient's plasma. These changes were not influenced by taking $300 \mathrm{mg}$ of aspirin per day. Flurbiprofen, a strong inhibitor of the release action of platelets, was tried. Within three hours of administering a single $25-\mathrm{mg}$ dose spontaneous aggregation could not be demonstrated. There was also complete inhibition of secondary aggregation in response to an ADP concentration of $5 \mathrm{mmol} / \mathrm{l}$ compared with 
the obvious aggregation with $0.1 \mathrm{mmol} / 1$ seen before treatment. This effect lasted for 12 hours, during which she was free of symptoms.

The patient was treated with flurbiprofen for three months, during which time her symptoms did not recur. Within a few days of discontinuing this treatment she complained of the same symptoms. Since then it has been decided to start long-term flurbiprofen treatment again and subsequently she has remained asymptomatic. Her gastrointestinal symptoms also disappeared. This suggests the possibility that the spontaneous platelet aggregation was also causing gut ischaemia.

\section{Comment}

The cause of spontaneous platelet aggregation in myeloproliferative and other disorders is unknown, but we suggest that when it fails to respond to treatment with aspirin flurbiprofen may be an effective alternative.

1 Vreeken, J, and Van Aken, W G, Lancet, 1971, 2, 1394.

2 Bierme, R, et al, Lancet, 1972, 1, 432.

3 Preston, F E, et al, British Medical fournal, 1974, 3, 548.

4 Scrobohaci, M L, Gunescu, G, and Orha, I, Thrombosis and Haemostasis, 1976, 36, 645.

5 Born, G V R, Nature, 1962, 194, 927.

(Accepted 5 Fanuary 1978)

Department of Haematology, Charing Cross Hospital, London W6 8RF

C POLITIS-TSEGOS, MD (Athens), honorary lecturer in haematology SYLVIA WOLFF, senior technician

S SHAW, MD, FRCPATH, consultant haematologist

T R MITCHELL, MRCPATH, senior lecturer in haematology

\section{Patients' responses to barium $x$-ray studies}

Several studies have suggested that patients often feel acute anxiety during diagnostic and technical procedures. Hoare and Hawkins ${ }^{1}$ found that an unacceptably large number of patients reported that endopscopy was unpleasant and stressful without sedation, and noted that careful discussion beforehand could be beneficial. This agreed with the findings of Johnson et al, ${ }^{2}$ who showed how a detailed explanation of the sensations and experience of endoscopy before the procedure reduced patients' anxiety. Another study by Johnson $e t a^{3}$ of children having a plaster-of-Paris cast removed also showed how previous explanation was associated with less anxiety.

This study aimed at seeing whether detailed information about the procedure and experiences of the patient before and during a barium meal or enema given on the day before one of these $x$-ray studies would affect their level of anxiety before and during the procedure.

\section{Methods and results}

Two similar studies were undertaken concurrently, one with patients having a barium meal for the first time and the other with those having a barium enema for the first time. These were performed in two London teaching hospitals with inpatients from all types of wards scheduled for either of these $x$-ray studies. Each of the samples of 58 patients having a barium meal and 70 having a barium enema was divided equally into experimental and control groups. In the former patients received a specially prepared explanation from one researcher, which took five minutes. Control patients received a visit from this researcher, who talked about unrelated topics for the same time. This researcher allocated patients alternately into experimental and control groups, while a second researcher tested their emotional responses, without knowledge of which group they were in. Selfreport measures were used to rate emotional reactions. A mood adjective check list used by Lishman ${ }^{4}$ was completed by patients on four occasions over a 24-hour period. These occasions were: $(a)$ as a baseline, early on the afternoon before the $\boldsymbol{x}$-ray investigation; $(b)$ late on the afternoon before the $x$-ray study, after the other researcher's visit, to assess any reactions to the explanation; $(c)$ half an hour before the $x$-ray study; $(d)$ within half an hour after the study, to describe feelings during it. Four anxiety scores were therefore obtained for each patient, the maximum score being 12. Experimental and control scores were compared at each of the four stages using the MannWhitney U test. ${ }^{5}$

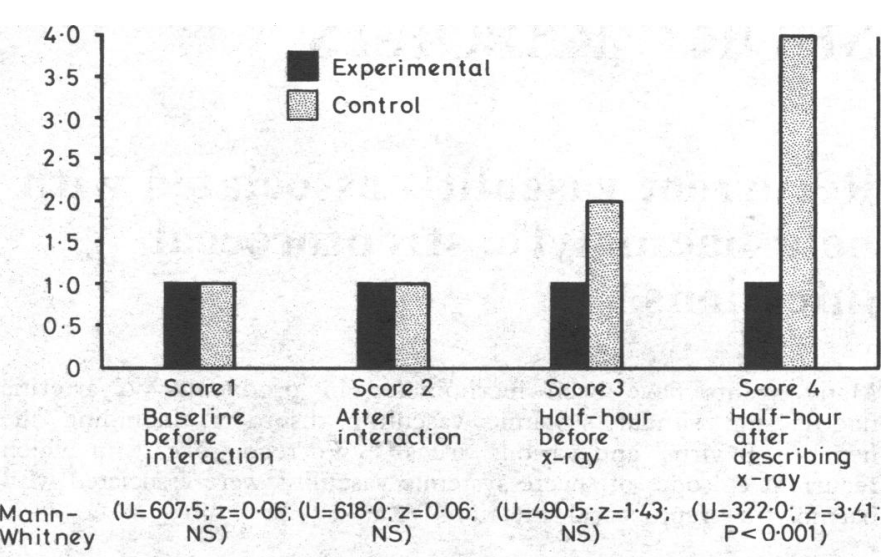

Median anxiety scores in barium-enema study (70 patients).

Patients having a barium meal reported low levels of anxiety before and 3 during this. There were no significant differences between experimental and control groups on any of the four anxiety scores. Patients having a barium os enema reported high levels of anxiety during this (see figure). Those who $\vec{N}$ had received the explanation were significantly less anxious than those in the control group during the $x$-ray study.

\section{Conclusion}

This study shows that explaining the procedure of a barium enema beforehand resulted in reduced anxiety. Barium meals were less stressful and explanation was also less effective in reducing anxiety.

Factors contributing to the stressfulness of a barium-enema study include waiting time in the $x$-ray department; moving about on the hard $x$-ray table; and the darkness and noise during screening. By far the most common complaint was the bowel preparation: soap enemata or the use of Dulcolax (bisacodyl) tablets produced equally painful results, and many patients were sleepless and exhausted before the investigation. Hence it seems important that doctors should recognise that the procedure is stressful and that prior explanation is beneficial.

This research was financed by the DHSS and supervised by Professor James Watson at Guy's Hospital, London. I thank all the hospital staff and patients who helped with this work.

1 Hoare, A M, and Hawkins, C F, British Medical fournal, 1976, 3, 20.

2 Johnson, J, Morrisey, J F, and Leventhall, H, Gastrointestinal Endoscopy, $1973,19,180$

${ }^{3}$ Johnson, J, Kirchoff, K J, and Endress, M P, Nursing Research, 1975, 24, 404.

4 Lishman, W A, Psychological Medicine, 1972, 2, 248.

5 Siegal, S, Non Parametric Statistics for the Behavioural Sciences. London, McGraw Hill, 1956.

(Accepted 5 fanuary 1978)

Department of Nursing Studies, Chelsea College, London SW1 JENIFER WILSON-BARNETT, PHD, DIPN, lecturer

\section{Severe envenomation from "harmless" pet snake}

We report the first recorded case of severe envenomation after a bite from Rhabdophis subminiatus. This snake, commonly known as the red-neck keel-back snake, is widely distributed in South-east Asia ${ }^{1}$ and is occasionally sold in pet shops in Britain. It has hitherto been considered to be non-venomous.

\section{Case report}

One of us (TMM) when a 24-year-old medical student, was bitten on the proximal phalanx of his left index finger by a $R$ subminiatus, kept as a pe 